\title{
Alterations in cortisol and interleukin- 6 secretion in patients with COVID-19 suggestive of neuroendocrine-immune adaptations
}

\author{
Maria P. Yavropoulou (10 ${ }^{1}$ - Maria G. Filippa ${ }^{1}$ - Aimilia Mantzou ${ }^{2} \cdot$ Fotinie Ntziora $^{1} \cdot$ Maria Mylona $^{1}$. \\ Maria G. Tektonidou ${ }^{1} \cdot$ Nikolaos I. Vlachogiannis $^{1} \cdot$ Dimitrios Paraskevis $^{3} \cdot$ Gregory A. Kaltsas $^{1}$ • \\ George P. Chrousos ${ }^{4} \cdot$ Petros P. Sfikakis ${ }^{1}$
}

Received: 2 November 2021 / Accepted: 11 December 2021 / Published online: 18 January 2022

(c) The Author(s), under exclusive licence to Springer Science+Business Media, LLC, part of Springer Nature 2022

\begin{abstract}
Purpose The beneficial effect of glucocorticoids in coronavirus disease (COVID-19) is established, but whether adrenal cortisol secretion is impaired in COVID-19 is not fully elucidated. In this case-control study, we investigated the diurnal free bioavailable salivary cortisol secretion in COVID-19 patients.

Methods Fifty-two consecutive COVID-19 patients—-before dexamethasone treatment in cases required—recruited between April 15 to June 15, 2021, (NCT04988269) at Laikon Athens University-Hospital, and 33 healthy age- and sex-matched controls were included. Diurnal salivary cortisol (8 a.m., 12, 6, and 10 p.m.), plasma adrenocorticotropin (ACTH) and aldosterone, and serum interleukin-6 (IL-6) and C-reactive protein (CRP) levels were assessed. Diurnal salivary dehydroepiandrosterone (DHEA) and IL-6 were also assessed in subgroups of patients.

Results Median CRP and IL-6 measurements were about sixfold higher in patients than controls (both $p<0.001$ ) Morning salivary cortisol levels did not differ between the two groups, but patients exhibited higher median levels of evening and nocturnal salivary cortisol compared to controls $[0.391(0.054,0663)$ vs. $0.081(0.054,0.243) \mu \mathrm{g} / \mathrm{dl}, p<0.001$ and $0.183(0.090,0.834)$ vs. $0.054(0.054,0.332) \mu \mathrm{g} / \mathrm{dl}, p<0.001$, respectively], resulting in higher time-integrated area under the curve (AUC) $(4.81 \pm 2.46$ vs. $2.75 \pm 0.810$, respectively, $p<0.001$ ). Circulating ACTH, DHEA, and aldosterone levels were similar in patients and controls. Serum IL-6, but not ACTH levels, was strongly correlated with nocturnal cortisol salivary levels $(\rho=0.555, p<0.001)$ in patients. Conclusions Increased evening and nocturnal but not morning cortisol secretion may occur in even clinically mild COVID19. In the context of acute viral infection (COVID-19), IL-6 may partially replace ACTH as a stimulus of the glucocorticoidsecreting adrenal zona-fasciculata without influencing the secretion of DHEA and aldosterone.

Clinical trial registration https://clinicaltrials.gov/ct2/show/NCT04988269?term=yavropoulou\&draw $=2 \&$ rank $=3$ (NC T04988269).
\end{abstract}

Keywords Diurnal salivary cortisol $\cdot$ Diurnal salivary dehydroepiandrosterone $\cdot$ Aldosterone $\cdot$ Adrenocorticotropin $\cdot$ SARS-CoV-2

These authors contributed equally: George P. Chrousos, Petros P. Sfikakis

Supplementary information The online version contains supplementary material available at https://doi.org/10.1007/s12020021-02968-8.

Maria P. Yavropoulou

myavropoulou@med.uoa.gr

1 1st Department of Propaedeutic and Internal Medicine, Medical School, National and Kapodistrian University of Athens, 11527 Athens, Greece

2 University Research Institute of Maternal and Child Health and Precision Medicine Medical School, National and Kapodistrian University of Athens, 11527 Athens, Greece

\section{Introduction}

In March 2020, acute infection of the respiratory tract caused by a novel coronavirus (severe acute respiratory

3 Department of Hygiene, Epidemiology and Medical Statistics, Medical School, National and Kapodistrian University of Athens, 11527 Athens, Greece

4 University Research Institute of Maternal and Child Health and Precision Medicine and UNESCO Chair on Adolescent Health Care, Medical School, National and Kapodistrian University of Athens, 11527 Athens, Greece 
syndrome coronavirus 2 [SARS-CoV-2]) was officially declared by the World Health Organization (WHO) a global pandemic, which has so far (October 29, 2021 [http:// covid19.who.int/]) led to 4.979.421 deaths. Although many patients infected with coronavirus disease (COVID-19) are asymptomatic or have mild symptoms [1], 10-15\% present with severe symptoms and may require invasive mechanical ventilation [2]. In the Randomized Evaluation of COVID19 Therapy (RECOVERY) study, administration of the synthetic glucocorticoid, dexamethasone reduced the death rate by about one-third in hospitalized COVID-19 patients [3]. Based on this evidence, glucocorticoid administration is currently considered as first-line treatment in hospitalized adults with COVID-19 (World Health Organization. Therapeutics and COVID-19: living guideline, 31 March 2021. No. updated Aug 25, 2021 WHO/2019-nCoV/therapeutics/ 2021.1. 2021; Accessed 1 November 2021).

In search of potential factors affecting the outcome of hospitalized COVID-19 patients, previous studies assessed adrenal cortex response by measuring serum cortisol, dehydroepiandrosterone (DHEA), and aldosterone levels, reporting, however, conflicting results [4-10].

Interleukin-6 (IL-6) is a pleiotropic proinflammatory cytokine that regulates the acute-phase inflammatory response and plays a central role in immune-mediated diseases, being a therapeutic target in various inflammatory conditions [11-13]. Several lines of evidence supported a potential prognostic role for IL-6 for severe outcomes in hospitalized COVID-19 patients, showing that high IL-6 serum levels at admission predicted the development of hypoxemia requiring hospitalization [14-17]. IL-6 may also affect the function of endocrine tissues including stimulation of the hypothalamic-pituitaryadrenal (HPA) axis in healthy volunteers [18] and patients with sepsis [19] and cancer [20-22].

Herein, we sought to investigate the adrenocortical response of patients with COVID-19 focusing on the diurnal secretion of free bioavailable salivary cortisol before any therapeutic administration of dexamethasone. We also assessed plasma levels of adrenocorticotropin (ACTH) and aldosterone and the diurnal secretion of DHEA in saliva. To test whether the magnitude of individual immune response in COVID-19 correlates with cortisol production, we also performed serum and diurnal salivary measurements of IL-6.

\section{Patients and methods}

\section{Participants}

This was a single-center case-control study. Adult consecutive patients with PCR-confirmed COVID-19, who presented to the Emergency Department of Laikon Athens UniversityHospital from April 15 to June 15, 2021, were considered eligible for the study. Patients with pre-existing hypoadrenalism or concurrent systemic glucocorticoid treatment, chronic kidney disease stage $3 \mathrm{~b}$ and above, medical history of cancer and systemic rheumatic diseases were excluded.

All patients were classified upon presentation according to NIH guidelines for classification of COVID-19 severity (https://www.covid19treatmentguidelines.nih.gov/overview/ clinical-spectrum/). Briefly, patients who presented with signs and symptoms of COVID-19 without evidence of pneumonia were considered to have mild disease status. Clinical and/or radiographic evidence of lower respiratory tract disease with oxygen saturation $\left(\mathrm{SpO}_{2}\right) \geq 94 \%$ on room air was classified as moderate disease status. Patients with pneumonia and $\mathrm{SpO}_{2}<94 \%$ on room air, classified as having severe disease status, were treated with dexamethasone on admission as per the standard care practice, and, therefore, were excluded from the study. Enrolled patients were followed-up for 4 weeks after COVID-19 diagnosis for possible progression from mild to moderate/severe or from moderate to severe disease status.

Age- and sex-matched apparently healthy volunteers (frequency matching), who did not have pre-existing hypoadrenalism or concurrent systemic glucocorticoid treatment, chronic kidney disease stage $3 \mathrm{~b}$ and above, medical history of cancer and systemic rheumatic disease, were also recruited from the Hospital personnel during the same time period and served as controls. Only volunteers with negative PCR test for SARS-CoV-2 and without history of a contact with a COVID-19 case at the time of the study were included.

All participants signed an informed consent and the procedures followed were in accordance with the 1975/83 Declaration of Helsinki. The study was approved by the scientific committee of Laikon Athens University-Hospital (Approval number E.L. 310) and was registered in ClinicalTrials.gov (NCT04988269).

\section{Sampling procedure and measurements}

Blood samples were obtained from patients and controls immediately upon recruitment at an unscheduled timepoint for the measurements of C-reactive protein (CRP), IL-6, ACTH, and aldosterone levels. During the next day, four salivary samples were obtained at prescheduled time points, namely, at 8 a.m., and 12-noon, 6, and 10 p.m. for free cortisol levels (for all participants). DHEA and IL-6 were measured in subgroups of 30 and 20 patients with available samples, respectively. Both subgroups were representativein terms of age and gender -of the whole group of patients. The participants were asked to refrain from eating and brushing their teeth for $1 \mathrm{~h}$ before the collection. Salivary samples for cortisol measurements were collected using the Salivette device (Sarstedt, Nümbrecht, Germany) and for DHEA and IL-6 measurements using passive drool method 
without a swab. The salivary samples were refrigerated after collection at $0-4{ }^{\circ} \mathrm{C}$. Within $24 \mathrm{~h}$ after saliva collection, the salivettes were centrifuged at $2400 \times g$ for $20 \mathrm{~min}$ at $4{ }^{\circ} \mathrm{C}$ and aliquots were stored at $-80^{\circ} \mathrm{C}$ until further cortisol analysis. Collection tubes for DHEA and IL-6 analysis were stored at $-80^{\circ} \mathrm{C}$.

Serum high sensitivity CRP (hsCRP) and plasma ACTH concentrations were measured using solid-phase, two-site chemiluminescence immunoassays on an IMMULITE 2000 Immunoassay System (Siemens Healthcare Diagnostics Products Ltd, UK). The analytical sensitivity for ACTH measurement was $5 \mathrm{pg} / \mathrm{mL}$ and for hsCRP measurement $0.1 \mathrm{mg} /$ $\mathrm{L}$. The intra and inter-assay coefficients of variation (CV) for ACTH ranged from $6.7 \%$ to $9.5 \%$ and from $6.1 \%$ to $10 \%$, respectively, and for hsCRP from 2.8 to $8.7 \%$ and from $3.1 \%$ to $8.7 \%$, respectively, depending on the sample concentration. Plasma aldosterone was assessed by a competitive enzyme immunoassay using ELISA DIAsource kit (DIAsourceImmunoAssays S.A., Belgium) and serum IL-6 was detected by a sandwich enzyme immunoassay using Quantikine ELISA kit (R\&D Systems, Minneapolis, MN, USA). The lower detection limit of DIAsource aldosterone assay was $15 \mathrm{pg} / \mathrm{mL}$ and the intra- and inter- assay CV laid between $4.5 \%$ and $8.7 \%$, respectively, depending on sample concentration. The lower detectable measurement of IL-6 was $0.70 \mathrm{pg} / \mathrm{mL}$ and the inter- and intra-assay precision ranged between 1.6 and 4.2 and from 3.3 to 6.4 , respectively.

For those samples with undetectable serum IL-6 levels we arbitrarily used the value of $0.1 \mathrm{pg} / \mathrm{mL}$, which was the lowest value in the analytical curve of the assay.

On the day of measurement, salivary samples were thawed, vortexed, and centrifuged at $1500 \mathrm{rpm}$ for $15 \mathrm{~min}$. Salivary cortisol was measured by an electrochemiluminescence immunoassay on the automated analyzer Cobas e411-Roche Diaghnostics (GmbH, Mannheim). The detection limit was $0.054 \mathrm{mcg} / \mathrm{dL}$ and the intra- and inter-assay CV were $6.1 \%$ and $11.8 \%$, respectively, at the concentration of $0.137 \mathrm{mcg} / \mathrm{dL}$.

Our laboratory has investigated the transferability of the reference range reported in the manufacturers' insert to our population by measuring saliva cortisol samples from 139 apparently healthy individuals aged 21 years and older at four time points during a day. Exclusion criteria were pregnancy, lactation, use of oral contraceptives and medication with cortisone/cortisol. The following reference values (95th percentile) were determined and no statistical difference was observed between males and females. Morning hours ( 6 a.m. to 12-noon): <0.78 mcg/dl; Afternoon hours (4-8 p.m.): $<0.24 \mathrm{mcg} / \mathrm{dl}$; Late night hours (10-12 p.m.); $0.21 \mathrm{mcg} / \mathrm{dl}$.

The concentrations of salivary DHEA and IL-6 were determined by a competitive enzyme immunoassay and a sandwich immunoassay, respectively (Salimetrics, USA). The functional sensitivity for DHEA measurements was reported by the manufacturer as $8.32 \mathrm{pg} / \mathrm{mL}$ and the intraand inter-assay $\mathrm{CV}$ ranged from $5.3 \%$ to $5.8 \%$ and from $7.9 \%$ to $8.5 \%$, respectively. The analytical sensitivity for IL-6 measurements was reported by the manufacturer as $0.07 \mathrm{pg} / \mathrm{mL}$ and the intra- and inter-assay $\mathrm{CVs}$ ranged from $3 \%$ to $10 \%$ and from $6 \%$ to $8 \%$, respectively.

\section{Statistics}

The Shapiro-Wilk test was used to assess for normality of distributions, and the data are presented as mean \pm standard deviation (SD) or median (min, max), as applicable.

Student's t-test for independent samples or the Mann-Whitney $U$-test was used for comparisons of continuous variables between patients and controls, as applicable. Univariate linear regression analysis was used for associations of disease status with the measurements of cortisol, DHEA, aldosterone, ACTH, and IL-6 levels. Multivariate linear regression analysis was used to adjust for differences in disease status within the COVID-19 patients' group.

Time-integrated daily cortisol and DHEA secretion were calculated employing the integrated area under the curve (AUC) using the trapezoidal method, as previously described [23]. All $p$ values are two-sided and a value of $p<0.05$ was considered statistically significant. Statistical analysis was performed using IBM SPSS Statistics for Windows, Version 26 (IBM SPSS Statistics for Windows, IBM Corporation, Armonk, NY, USA) and GraphPad Prism for Windows, Version 7 (GraphPad Software, San Diego, CA, USA).

The proportion of missingness was small (less than $6 \%$ of the measurements), therefore no sensitivity analysis for selection bias or unmeasured confounding in missing data was performed.

\section{Results}

\section{Characteristics of COVID-19 patients}

Fifty-two patients with COVID-19 and 33 controls matched for age and sex were finally included in the analysis (Fig. 1). Patients who presented only with symptoms such as fever, cough, sore throat, malaise, headache, muscle pain, nausea, vomiting, diarrhea, and loss of taste and/or smell, were classified as mild disease $(n=30)$, while patients presenting with additional clinical and/or radiographic evidence of lower respiratory tract infection with $\mathrm{SpO}_{2} \geq 94 \%$ on room air were classified as having moderate disease $(n=22)$. None had severe disease status at the time of first evaluation. The majority of patients had a favorable clinical course during the next 4 weeks (mild-stable or moderate-stable), while 8 patients of the 22 who were classified as moderately ill progressed to severe clinical status and required 
Fig. 1 Flow diagram of finally included COVID-19 patients

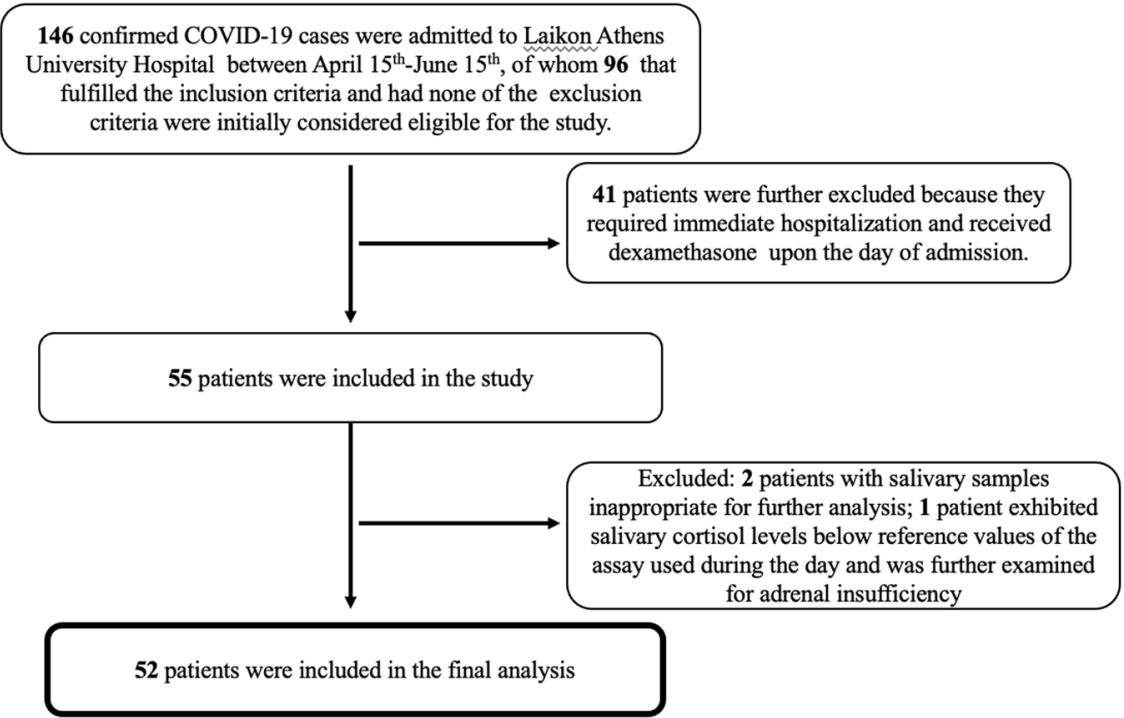

hospitalization (moderate-progressed). Among those 8 patients with moderate-progressed disease, 3 patients required immediate hospitalization and thus blood and salivary samples were obtained at the hospital, while the rest were treated as outpatients, but required hospitalization within a week after the first admission.

Age and BMI were similar between patients and controls $(44.8 \pm 14.2$ years vs. $47.6 \pm 12.8$ years, $p=0.359$ and $25.9 \pm 5.1 \mathrm{~kg} / \mathrm{m}^{2}$ vs. $\left.24.8 \pm 3.8 \mathrm{~kg} / \mathrm{m}^{2}, \mathrm{p}=0.283\right)$. Diabetes mellitus type $2(5.8 \%)$, hypertension (13.5\%), and hypothyroidism $(13.5 \%)$ were the most common comorbidities in COVID-19 patients. A total of $31 \%$ of patients and $19 \%$ of controls were current smokers $(p=0.397)$. Table 1 summarizes the demographic and clinical characteristics of COVID-19 patients stratified by disease status and course as mild-stable $(n=30)$, moderate-stable $(n=14)$, and moderate-progressed $(n=8)$, and controls. As expected, patients with a mild-stable disease course were significantly younger than the remaining patients, and had lower BMI compared to patients with moderate-stable disease course (Table 1). Also as expected, the median serum measurements of CRP were about sixfold higher in COVID-19 patients than controls $[5.6(0.05,90.1) \mathrm{mg} / \mathrm{L}$ vs. $0.9(0.1$, 9.0) $\mathrm{mg} / \mathrm{L}, p<0.001)$. Serum IL-6 levels were detectable in $7(21 \%)$ controls, and in $46(88 \%)$ patients and the median serum measurements were also about sixfold higher in COVID-19 patients than controls $[4.5(0.1,46.6) \mathrm{pg} / \mathrm{mL} v s$. $0.1(0.1,15.1) \mathrm{pg} / \mathrm{mL} p<0.001$, respectively), being much higher in patients with pneumonia than in those without (Table 1). Individual serum CRP levels were strongly correlated with the corresponding serum IL-6 levels in COVID-19 patients $(\rho=0.564, p<0.001$ (Supplementary Fig. 1).

\section{Diurnal cortisol secretion in COVID-19 patients}

Salivary cortisol levels in the morning (8 a.m.) were similar in COVID-19 patients and controls, whereas no significant differences were evident between patient subgroups (Table 1). All controls and most of the COVID-19 patients (46/52, $88 \%$ ) exhibited the expected gradual decrease of salivary cortisol during the day, with the lowest measurements obtained during evening and nocturnal sampling (Fig. 2a, b). However, as shown in Fig. 2c daily cortisol secretion was clearly increased in patients with either mild-stable, moderate-stable, or moderate-progressed COVID-19 compared to that of controls.

Except for the morning measurements, all other cortisol measurements during the day were significantly higher in COVID-19 patients than in controls (Table 1). While evening and nocturnal cortisol levels decreased by $80 \%$ and $83 \%$, respectively, in controls (Fig. 2b), COVID-19 patients displayed much lower mean respective decreases, respectively 37 and 54\% (both $p<0.001$ ). Overall, time-integrated daily cortisol secretion, as assessed by the AUC, was almost twofold higher in COVID-19 patients than controls (AUC: $4.81 \pm 2.46$ vs. $2.75 \pm 0.810$, respectively, $p<0.001$ ), being lower, albeit non-significantly, in patients with mild disease than in the remaining patients (Fig. 2c). Thus, the presence of pneumonia was not related to the increased daily cortisol secretion in these patients $(\beta=0.132,95 \% \mathrm{CI} ;-0.8,2)$. Notably, the highest AUC of cortisol was observed in a patient with moderate-stable COVID-19 (individual value 10.86), which was almost double compared to the highest AUC observed among controls (individual value 4.73). 
Table 1 Demographic, clinical and laboratory characteristics of COVID-19 patients and controls

\begin{tabular}{|c|c|c|c|c|c|}
\hline \multirow[t]{2}{*}{ Parameters } & \multicolumn{3}{|c|}{ COVID-19 patients (Cases) ${ }^{\#}$} & \multirow{2}{*}{$\begin{array}{l}\text { Healthy controls } \\
(n=33)\end{array}$} & \multirow[t]{2}{*}{$p$ value } \\
\hline & $\begin{array}{l}\text { Mild-stable } \\
(n=30)\end{array}$ & $\begin{array}{l}\text { Moderate-stable } \\
(n=14)\end{array}$ & $\begin{array}{l}\text { Moderate-progressed } \\
(n=8)\end{array}$ & & \\
\hline Age (yrs), & $39.0 \pm 14.1$ & $53.4 \pm 10.3$ & $51.4 \pm 10.1$ & $47.6 \pm 12.8$ & $0.002^{a, b, c}$ \\
\hline Males $(n, \%)$ & $17(56.7 \%)$ & $7(50 \%)$ & $4(50 \%)$ & $19(57.6 \%)$ & 0.952 \\
\hline Days from positive PCR test & $4(2,13)$ & $4(2,15)$ & $3(2,9)$ & $\mathrm{n} / \mathrm{a}$ & 0.743 \\
\hline BMI $\left(\mathrm{kg} / \mathrm{m}^{2}\right)$ & $24.7 \pm 5.0$ & $29.6 \pm 4.5$ & $25.0 \pm 3.7$ & $24.8 \pm 3.8$ & $0.014^{\mathrm{a}, \mathrm{d}, \mathrm{e}}$ \\
\hline $\operatorname{DMT} 2(n, \%)$ & $1(3.3 \%)$ & $2(14.3 \%)$ & 0 & $1(4.0 \%)$ & 0.381 \\
\hline Smoking $(n, \%)$ & $8(36.4 \%)$ & $3(25 \%)$ & $2(28.6 \%)$ & $5(19.2 \%)$ & 0.612 \\
\hline Hypertension $(n, \%)$ & $2(6.7 \%)$ & $4(28.6 \%)$ & $1(12.5 \%)$ & $1(4.0 \%)$ & 0.088 \\
\hline $\begin{array}{l}\text { *Serum CRP levels } \\
\mathrm{RV} \text { of the assay: }<8 \mathrm{mg} / \mathrm{L}\end{array}$ & $3.0(0.1,17.6)$ & $17.0(0.5,90.2)$ & $18.3(0.3,51.0)$ & $0.9(0.1,9.1)$ & $<\mathbf{0 . 0 0 1}^{\mathrm{a}, \mathrm{b}, \mathrm{e}, \mathrm{f}}$ \\
\hline $\begin{array}{l}\text { *Serum Il-6 levels } \\
\text { RV of the assay: }<3.13 \mathrm{pg} / \mathrm{ml}\end{array}$ & $1.8(0.1,30.9)$ & $7.0(1.6,32.2)$ & $14.7(3.0,46.6)$ & $0.1(0.1,15.1)$ & $<\mathbf{0 . 0 0 1}^{\mathrm{a}, \mathrm{b}, \mathrm{c}, \mathrm{e}, \mathrm{f}}$ \\
\hline $\begin{array}{l}\text { *Plasma ACTH levels } \\
\text { RV of the assay: morning 9-52 pg/ml; afternoon } \\
5-30 \mathrm{pg} / \mathrm{ml}\end{array}$ & $16.1(5,56)$ & $19.1(5,31)$ & $14.0(5,40)$ & $16.3(5,47)$ & 0.642 \\
\hline $\begin{array}{l}\text { *Plasma Aldosterone levels } \\
\text { RV of the assay: } 40-310 \mathrm{pg} / \mathrm{ml}\end{array}$ & $127(60,367)$ & $85(32,523)$ & $206(33,275)$ & $140(42.4,438)$ & 0.398 \\
\hline $\begin{array}{l}\text { Morning (8 a.m.) cortisol measurement in the } \\
\text { saliva } * * \mathrm{RV} \text { of the assay: }<0.78 \mathrm{mcg} / \mathrm{dl}\end{array}$ & $0.493(0.09,1.122)$ & $0.506(0.281,1.170)$ & $0.828(0.199,1.060)$ & $0.533(0.285,1.020)$ & 0.573 \\
\hline $\begin{array}{l}\text { Mid-Day (12-noon) cortisol measurement in } \\
\text { the saliva** } \\
\mathrm{RV} \text { of the assay: }<0.78 \mathrm{mcg} / \mathrm{dl}\end{array}$ & $0.313(0.076,1.164)$ & $0.321(0.095,0.421)$ & $0.310(0.054,0.663)$ & $0.188(0.083,0.453)$ & $0.002^{c, e, f}$ \\
\hline $\begin{array}{l}\text { Evening (6 p.m.) cortisol measurement in the } \\
\text { saliva*** } \\
\text { RV of the assay: }<0.24 \mathrm{mcg} / \mathrm{dl}\end{array}$ & $0.149(0.062,0.906)$ & $0.228(0.121,0.892)$ & $0.391(0.054,1.010)$ & $0.081(0.054,0.243)$ & $<\mathbf{0 . 0 0 1}^{\mathrm{ce}, \mathrm{f}}$ \\
\hline $\begin{array}{l}\text { Nocturnal }(10 \mathrm{pm}) \text { cortisol measurement in } \\
\text { the saliva** } \\
\mathrm{RV} \text { of the assay: }<0.21 \mathrm{mcg} / \mathrm{dl}\end{array}$ & $0.116(0.054,1.010)$ & $0.234(0.054,1.410)$ & $0.183(0.090,0.834)$ & $0.054(0.054,0.332)$ & $<0.001^{\mathrm{c}, \mathrm{e}, \mathrm{f}}$ \\
\hline
\end{tabular}

Values are shown as mean $\pm \mathrm{SD}$, or median (min, max), as applicable. Categorical data are shown as absolute number (valid percentage). Twotailed Fisher's exact test was used to compare categorical data

One way ANOVA or Kruskal-Wallis H test with Bonferroni or Dunn's Post Hoc tests, respectively, were performed for multiple comparisons between groups, as applicable

$p$ values that are statistically significant are shown in bold

$P C R$ polymerase chain reaction, yrs years, $B M I$ body mass index, $D M T 2$ diabetes mellitus type $2, R V$ reference values, $C R P C$ - reactive protein, IL-6 interleukin-6, ACTH adrenocorticotropin

\#Classification based on disease severity (https://www.covid19treatmentguidelines.nih.gov/overview/clinical-spectrum/), and the clinical outcome of patients in the 4 weeks follow-up period of the study

*Blood sampling was performed at the day of recruitment (for both patients and controls) at unscheduled time points

**Saliva sampling was performed at the next day at prescheduled time points within the day (namely, 8 a.m.; 12-noon; 6 p.m.; 10 p.m.)

${ }^{\mathrm{a}} p<0.05$ for comparisons between patients with mild-stable and moderate-stable disease

${ }^{\mathrm{b}} p<0.05$ for comparisons between patients with mild-stable and moderate-progressed disease

${ }^{c} p<0.05$ for comparisons between patients with mild-stable disease and healthy controls

${ }^{\mathrm{d}} p<0.05$ for comparisons between patients with moderate-stable and moderate-progressed disease

${ }^{\mathrm{e}} p<0.05$ for comparisons between patients with moderate-stable disease and healthy controls

${ }^{\mathrm{f}} p<0.05$ for comparisons between patients with moderate-progressed disease and healthy controls

\section{ACTH and aldosterone levels and diurnal DHEA secretion in COVID 19}

Plasma measurements of both ACTH and aldosterone upon presentation of COVID-19 patients were similar to those of controls, irrespectively of disease status (Table 1). As also shown in Table 1, 7 patients and one control subject were hypertensive. None was under treatment with aldosterone or angiotensin-converting enzyme inhibitors, whereas 5 patients ( 2 with a mild-stable disease course; 2 with a moderate-stable disease course; and 1 with a moderate -progressed disease course) and the control subject were treated with angiotensin receptor blockers (ARBs) (olmesartan, valsartan and telmisartan). When we repeated the analysis excluding the participants that were treated with ARBs, aldosterone levels again did not differ between the COVID -19 patients and controls $[118(31.9,523)$ vs. 140 $(42,438) \mathrm{pg} / \mathrm{ml}, p=0.673]$, nor between patients with a different disease status $(p=0.815$, assessed by Kruskal-Wallis $H$ test with Dunn's Post HocTest). 

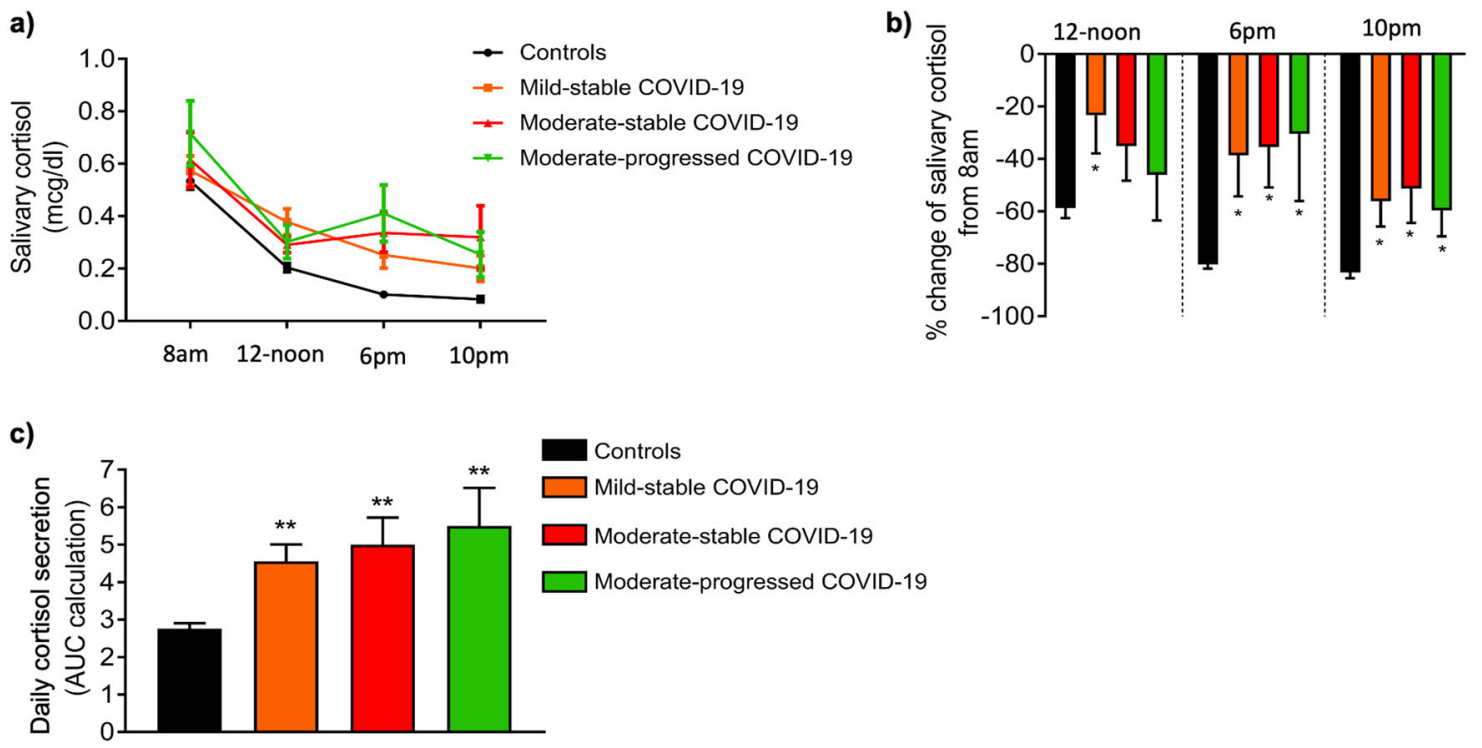

Fig. 2 Diurnal variation of salivary cortisol levels in COVID-19 patients, stratified by disease status, and healthy controls. a Cumulative salivary cortisol levels within each group, $\mathbf{b} \%$ change of salivary cortisol from 8 a.m., c time-integrated daily cortisol secretion (calculated as the area under the curvel). COVID-19 patients are classified according to the severity of the disease (https://www.covid19trea tmentguidelines.nih.gov/overview/clinical-spectrum/) and their clinical course during the 4-week follow-up period of the study as mild-stable, moderate-stable, and moderate-progressed. Values are shown as mean

Next morning salivary DHEA measurements were comparable in 30 representative COVID-19 patients and their matched controls (Supplementary Fig. 2a). In contrast to the increased time-integrated daily cortisol secretion, the sequential measurements of DHEA in saliva were similar in COVID19 patients, irrespectively of disease status, and controls (Supplementary Fig 2a). As a result, daily DHEA secretion did not differ between COVID-19 patients and controls [AUC for DHEA: $1956(328,6210)$ vs. $1546(116,6512), p=0.736)$ (Supplementary Fig. 2b) However, when we calculated the DHEA/cortisol ratio, at the 4 different time points during the day; 12-noon, 6 p.m., and 10 p.m. DHEA/cortisol ratios were significantly lower in COVID-19 patients compared to controls $(p<0.05)$, probably reflecting the higher salivary cortisol levels at these time points (Fig. 3a). Similar to salivary cortisol levels, however, no significant differences were observed between patients with a mild-stable and moderate-stable or moderate-progressed disease course (Fig. 3b).

\section{IL-6 serum levels correlate with daily cortisol secretion in COVID-19}

Serum IL-6 levels measured upon presentation were strongly correlated with the next day evening $(r h o=0.412$, $p=0.004)$ and nocturnal (rho $=0.555, p<0.001)$, but not with morning $($ rho $=0.219, p=0.126)$ or mid-day $($ rho $=$ \pm SEM, $* p<0.05$, comparisons were performed with the morning values of salivary cortisol (8 a.m.). One-way repeated measures ANOVA with Bonferroni post hoc test was used for multiple comparisons between different time points of salivary cortisol within each group. ${ }^{* *} p<0.05$, comparisons were performed with the control group, using Kruskal-Wallis followed by Dunn's multiple comparison test (comparing every group against the controls). AUC area under the curve

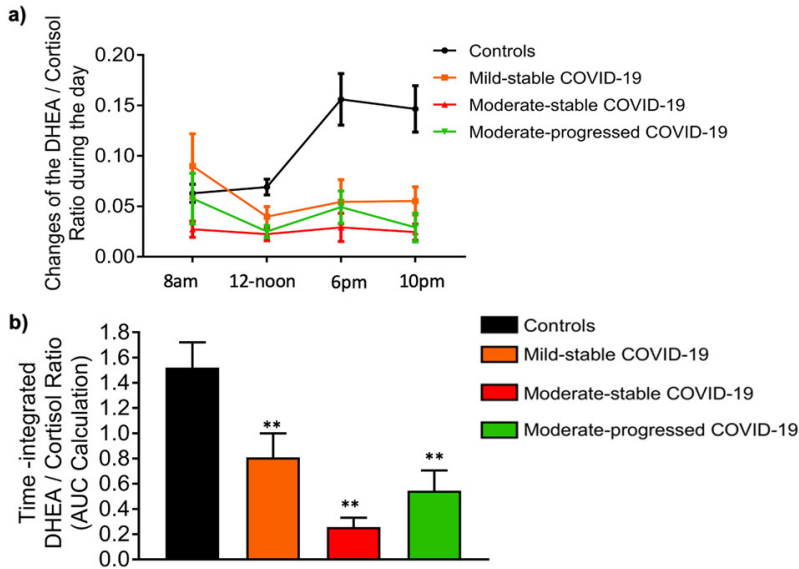

Fig. 3 Changes in the DHEA/Cortisol ratio during the day in COVID19 patients, stratified by disease status, and healthy controls. a Cumulative DHEA/Cortisol ratio within each group, b Timeintegrated changes of DHEA/Cortisol ratio during the day (calculated as the area under the curvel). COVID-19 patients are classified according to the severity of the disease (https://www.covid19trea tmentguidelines.nih.gov/overview/clinical-spectrum/) and their clinical course during the 4-week follow-up period of the study as mild-stable, moderate-stable, and moderate. $* * p<0.05$, comparisons were performed with the control group, using Kruskal-Wallis followed by Dunn's multiple comparison test (comparing every group against the controls). AUC, area under the curve

0.127, $p=0.374$ ) salivary cortisol levels (Fig. 4). In 20 representative COVID-19 patients, we also estimated the parallel diurnal IL-6 levels in saliva and found that increased 


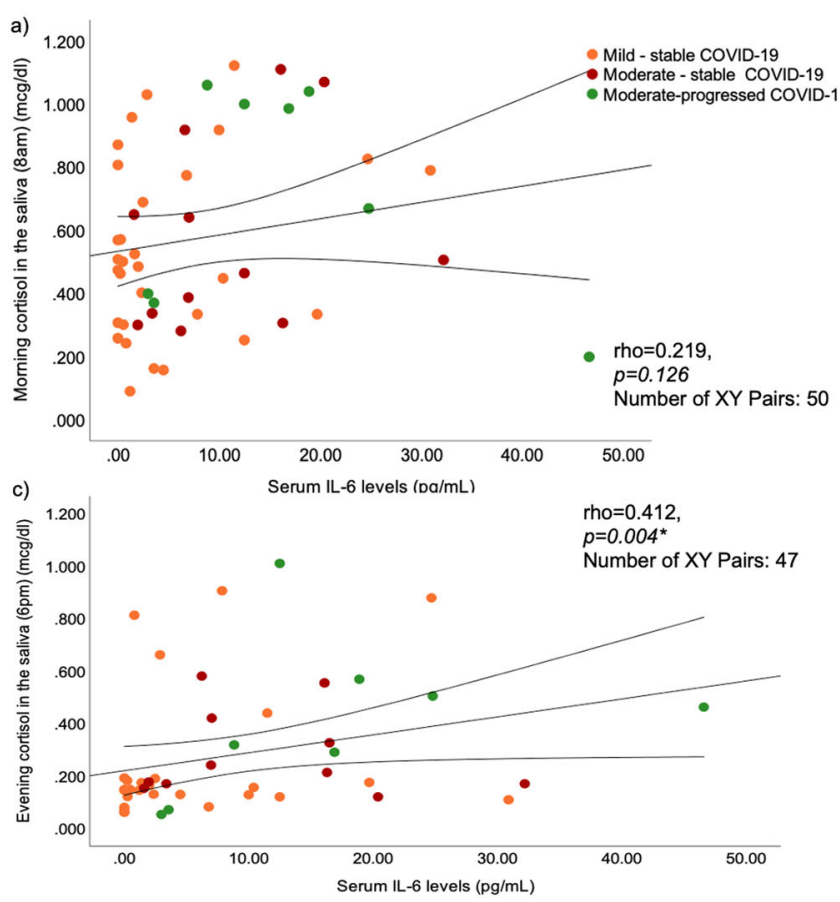

Fig. 4 Correlations of serum IL-6 levels measured on the day of recruitment with salivary cortisol levels measured during the next day in COVID-19 patients: a morning (8 a.m.), b mid-day (12-noon), c evening ( 6 p.m.), and d night (10 p.m.). Only the evening and night correlations were significant. COVID-19 patients are classified according to the severity of the disease (https://www.covid19trea

rates of IL-6 secretion were sustained in all COVID-19 patients tested from morning to night (Supplementary Fig. 3).

Collectively, individual serum IL-6 levels upon COVID-19 patients' presentation correlated with next day time-integrated daily cortisol secretion $(\mathrm{rho}=0.373$, $p=0.011$.

No associations were noted between individual serum IL-6 levels and concomitant plasma ACTH $($ rho $=0.167$, $p=0.242$ ) or plasma aldosterone ( $\mathrm{rho}=0.091, p=0.529)$ concentrations in COVID-19 patients. Likewise, serum IL-6 levels did not correlate with the next day timeintegrated daily DHEA secretion measured in saliva (rho $=-0.025, p=0.904)$, or the time-integrated changes of the DHEA/cortisol ratio during the day (calculated as AUC; rho $=-0.157, p=0.474)$, nor with the DHEA/ cortisol ratio at the different time points measured (data not shown).

Finally, no correlations were observed between plasma ACTH levels and the next day salivary cortisol measurements (Fig. 5), the time-integrated daily cortisol (rho $=$ $-0.048, p=0.749$ ) and DHEA secretion (rho $=-0.009$, $p=0.964$ ), or with the concomitantly measured plasma aldosterone levels (rho $=0.171, p=0.235$ ) in COVID-19 patients.

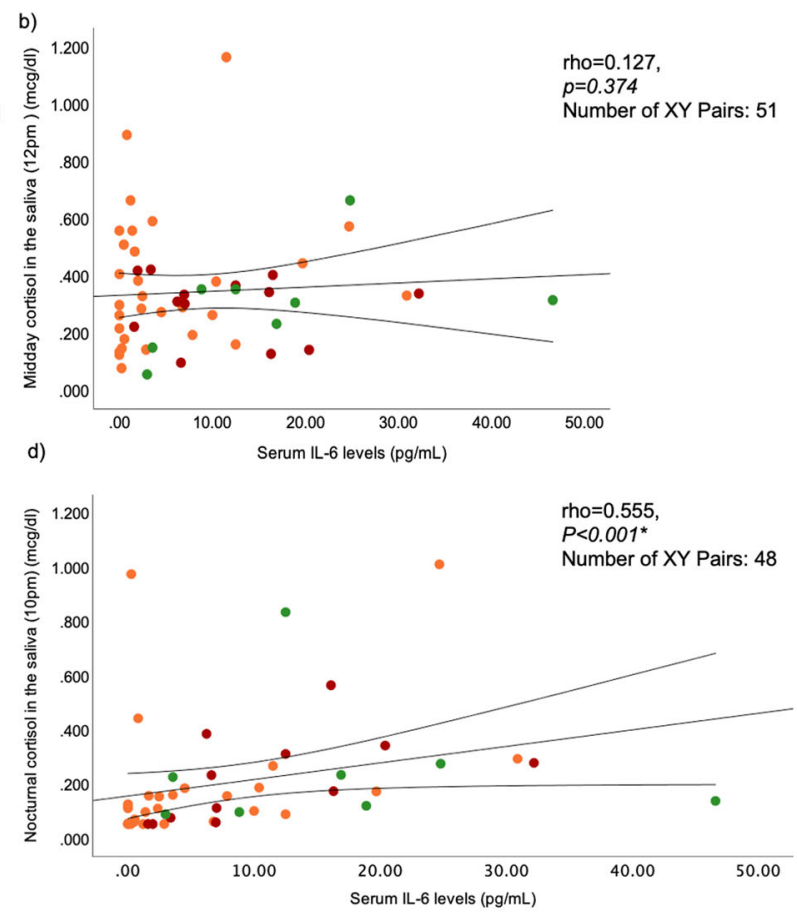

tmentguidelines.nih.gov/overview/clinical-spectrum/), and their clinical course during the 4-week follow-up period of the study, as mildstable, moderate-stable, and moderate-progressed. Spearman's rank correlation coefficient was used for associations between the parameters tested. ${ }^{*} p<0.05$ is considered statistically significant. IL-6, interleukin-6

\section{Discussion}

Our results show that the circadian rhythm of free bioavailable cortisol, is blunted in patients with even mild or moderate COVID-19, with higher evening and nocturnal cortisol levels, while salivary morning cortisol levels remain unaltered. These findings support our hypothesis, that increased daily, and especially evening cortisol secretion, is mostly driven by hypersecretion of IL- 6 in the context of the acute viral infection of COVID-19, in line with earlier findings suggestive of IL-6 acting as an ACTH-like stimulus of the glucocorticoid-secreting adrenal zona fasciculata [24, 25].

DHEA, and aldosterone levels, on the other hand, are not altered in patients with COVID-19, suggesting that a direct cytopathic effect of COVID-19 in the adrenal cortex [26-30] remains questionable, at least in patients with mild and moderate disease.

In addition, despite significant differences in IL-6 levels between COVID-19 patients with mild and moderate disease status, the increased daily cortisol secretion was not associated with the clinical disease status.

Tan et al. have recently reported that serum morning cortisol concentrations in patients with COVID-19 were higher than in those with similar symptoms in whom testing 
a)
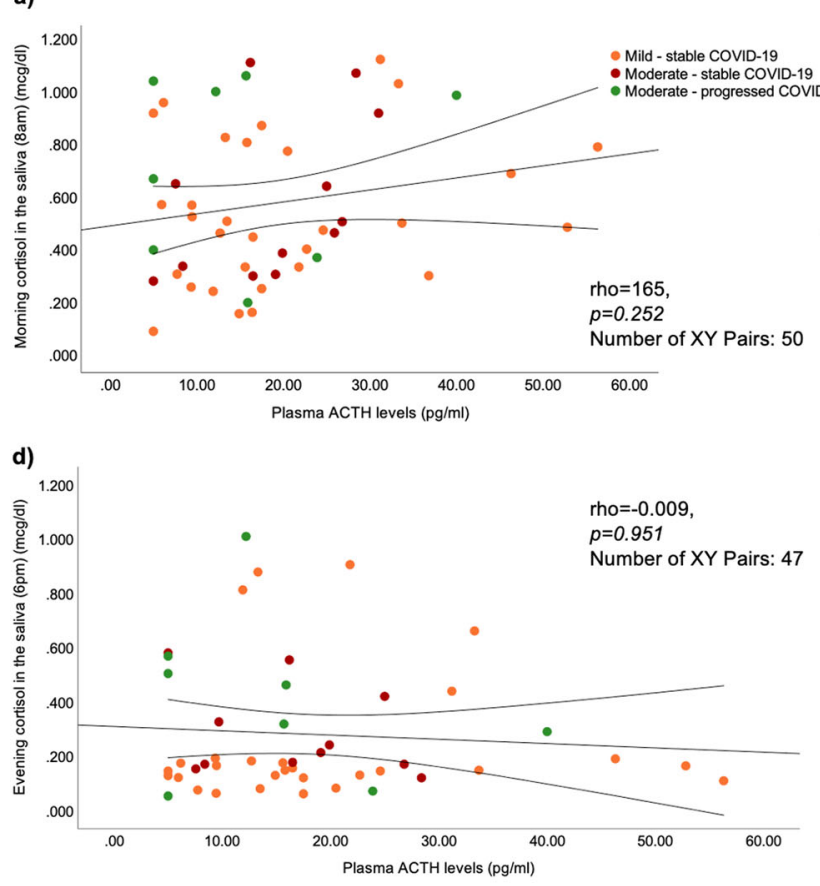

Fig. 5 Correlations of plasma ACTH measured on the day of hospital admission (Day 1) with salivary cortisol levels measured during the next day (Day 2) in COVID-19 patients: a morning (8 a.m.), b midday (12-noon), c evening (6 p.m.), and d night (10 p.m.). Correlations were not statistically significant. COVID-19 patients are classified

for the virus was negative [4]. In multivariate analysis, doubling of serum cortisol was associated with a $42 \%$ increase in mortality, after adjusting for age, comorbidities, and other laboratory tests [4], although not with disease severity [31]. Since $80-90 \%$ of circulating cortisol in human serum is protein-bound, changes in the binding proteins commonly found in critically ill patients [32] may falsely increase or decrease serum total cortisol measurements. In addition, considering that both ACTH and cortisol are pulsatile in critical illness [33], static assessments of serum total cortisol to predict mortality risks for COVID-19 patients should be interpreted with caution [34]. Nevertheless, our results suggest that morning total cortisol levels may not be of prognostic significance at least in mild to moderate COVID-19 disease, whereas increased daily cortisol secretion, particularly at the late hours of the day, is more likely to reflect high IL-6 levels, which is a wellknown robust prognostic biomarker in COVID-19 [35].

Alzahrani et al. reported lower cortisol and ACTH levels in COVID-19 patients with more severe disease vs. those with milder disease status, suggesting a direct link between COVID-19 infection and impaired glucocorticoid response, but the sample size was small $(n=28)$ and the majority of patients had relatively mild disease [5]. Our results do not support a COVID-19-associated direct insult on the HPA axis or on the adrenal cortex since we found no differences b)

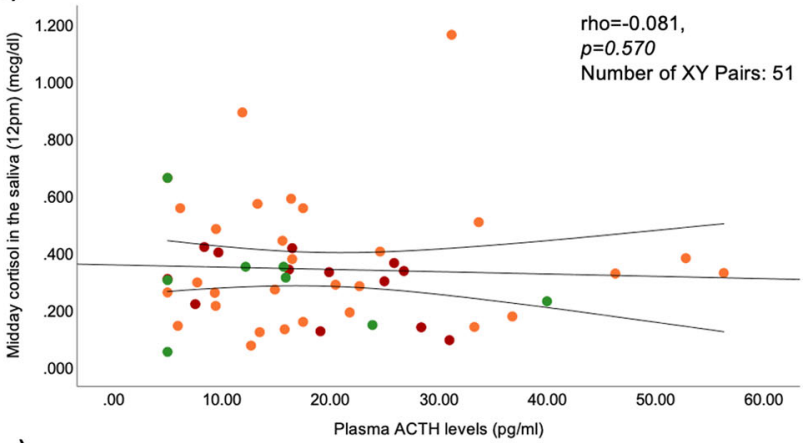

c)

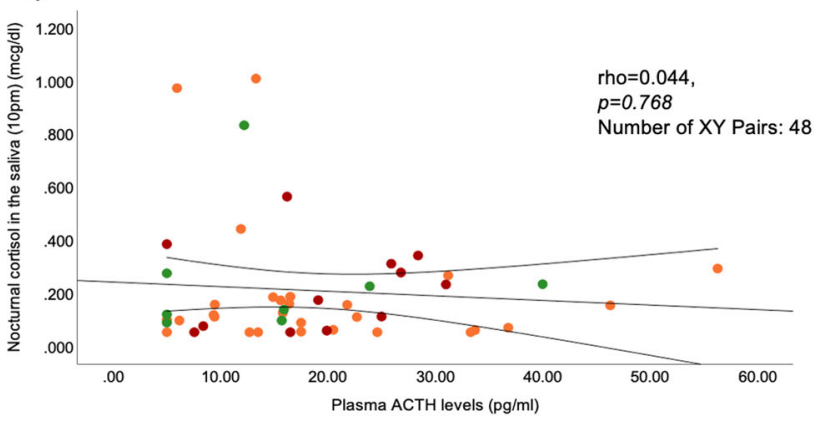

according to the severity of the disease (https://www.covid19trea tmentguidelines.nih.gov/overview/clinical-spectrum/), and their clinical course during the 4-week follow-up period of the study. Spearman's rank correlation coefficient was used for associations between the parameters tested. ACTH, adrenocorticotropin

in the circulating levels of ACTH, aldosterone and DHEA, between COVID-19 patients and controls. Serum cortisol levels were also assessed in 40 COVID-19 patients admitted at intensive or sub-intensive care unit in a recent study that evaluated the effects of SARS-CoV-2 on thyroid functioning tests (TFTs). In this study, although the majority of COVID-19 patients had TFTs within normal range, lower TSH and FT3 levels were inversely correlated with higher serum cortisol levels and higher IL-6 levels. In line with our data, the authors suggested that transient reductions of TFTs in COVID-19 probably reflect a direct or indirect effect (through increased cortisol) of IL-6, rather than a destructive thyroiditis due to SARS-CoV-2 infection [10].

Previous experimental studies in mice have shown a stimulatory effect of IL-6 on cortisol production by the adrenal cortex in the absence of corticotropin-releasing hormone [36], in cytomegalovirus-infected mice [37], as well as in murine colitis [38]. In humans IL-6 may stimulate cortisol release directly on the level of the adrenal gland in long-term stress situations [25], and the same may also apply in chronic inflammatory diseases, although direct evidence is still lacking [39]. Protein expression of IL-6 and IL-6 receptor has been detected in primary cultures of human adrenal cells depleted of macrophages (CD68-positive cells) predominantly in the zona reticularis but also in the inner zona fasciculata and in single cells within the zona 
glomerulosa and the medulla [24]. In this study, IL-6 was shown to induce adrenal steroidogenesis in vitro in a time and dose-dependent manner in the absence of macrophages, showing that IL-6 is a long-term stimulator of steroidogenesis but with no acute effects [24]. However, the hypothesis that IL-6 may partially replace ACTH when there is an acute requirement for increased cortisol secretion, as supported by these results in Covid-19, has not been fully elucidated. Along this line, our results may partly explain the lack of beneficial effect of the anti-IL-6 monoclonal antibody tocilizumab in COVID-19 patients who were not concurrently treated with dexamethasone, demonstrated in some studies. For example, in a multicenter, randomized, open-label study conducted in Brazil, where only $7 \%$ of the enrolled patients were receiving concomitant corticosteroid treatment, administration of tocilizumab in severe COVID-19 resulted to increased mortality at 15 days of treatment, leading to early termination of the trial [40]. Therefore, although a speculation, this implies that neutralization of IL- 6 activity by tocilizumab could aggravate the severity of the disease in cases not already treated with dexamethasone, by preventing endogenous cortisol production required in the context of highgrade inflammation.

Most of our COVID 19 patients, exhibited the expected gradual decrease of salivary cortisol during the day, underlining the preservation of the circadian rhythm of cortisol despite the need for increased production. Blunted circadian cortisol rhythm in humans has been observed in cancer, chronic stress, and depression [41], but data are scarce in acute viral infection. In particular, early mortality has been shown among patients with metastatic breast cancer [42], lung cancer [43], and epithelial ovarian cancer [44] with relatively "flat" rhythms, pointing out the vital role of normal diurnal cortisol variation in the endogenous control of inflammation.

The limitations of our study, besides its cross-sectional design and the rather small patient number, include: (i) lack of assessment of stress in our patients, which could contribute to IL-6 induction and (ii) exclusion, as per study design, of patients with severe COVID-19 infection who were treated with dexamethasone, which did not allow us to evaluate the overall effect of cytokine storm in adrenal response. In addition, 3 patients required immediate hospitalization while the rest were treated as outpatients at the time of blood and salivary samples collection, and this could suggest a different impact of stress between those patients, but the number was small, accounting to less than $6 \%$ of the study population. Furthermore, antihypertensive treatment with ARBs could interfere with the aldosterone measurements in our study, however, less than $10 \%$ of the COVID-19 patients and only one of the control subjects were treated with ARBs.
Strengths of the study are i) the assessment of diurnal production of both cortisol and DHEA, ii) the use of salivary samples which reflect the free locally available steroid hormone in target tissues and avoid the interference of altered binding proteins in the assay [45, 46] and iii) the inclusion of an age- and sex-matched apparently healthy control group.

To conclude, our results suggest that overproduction of IL-6 in the context of an acute viral infection-induced activation of the immune system, as in COVID-19, may partly replace the role of ACTH as a stimulus to release the required extra cortisol by the adrenal cortex; this being more evident at the late hours of the day even in patients with mild disease. Increased evening and nocturnal salivary but not morning cortisol levels may be considered a more reliable biomarker of disease severity in the context of acute SARS-CoV-2 infection. Finally, overproduction of IL-6 does not seem to affect the zonae glomerulosa and reticularis, producing mineralocorticoids and androgens, respectively, in the context of mild-moderate acute viral infection.

Acknowledgements We gratefully acknowledge the assistance and support of the medical personnel of the 1st Department of Propaedeutic and Internal Medicine, in particular Dr. Evangelia Tzeravini, Dr. Tsakanikas Aristeidis, Dr. Konstantinos Magganas, Dr. Ioannis Legakis, and Dr. Marina Tsoli.

Funding This work was supported by ELKE grants from the National and Kapodistrian University of Athens (PP Sfikakis). NIV has received a scholarship for doctoral studies by A. Onassis Public Benefit Foundation.

\section{Compliance with ethical standards}

Conflict of interest The authors declare no competing interests.

Publisher's note Springer Nature remains neutral with regard to jurisdictional claims in published maps and institutional affiliations.

\section{References}

1. O.E. Tsitsilonis, D. Paraskevis, E. Lianidou, E. Terpos A. Akalestos, V. Pierros et al. SARS-CoV-2 infection is asymptomatic in nearly half of adults with robust anti-spike protein receptorbinding domain antibody response. Vaccines 9, 1-12 (2021)

2. Z. Wu, J.M. McGoogan, Characteristics of and important lessons from the coronavirus disease 2019 (COVID-19) outbreak in China: summary of a report of 72314 cases from the chinese center for disease control and prevention. JAMA 323, 1239-42 (2020)

3. R.C. Group, P. Horby, W.S. Lim, J.R. Emberson, M. Mafham, J. L. Bell et al. Dexamethasone in Hospitalized Patients with Covid19. N. Engl. J. Med. 384, 693-704 (2021)

4. T. Tan, B. Khoo, E.G. Mills, M. Phylactou, B. Patel, P.C. Eng et al. Association between high serum total cortisol concentrations and mortality from COVID-19. Lancet Diabetes Endocrinol. 8, 659-60 (2020)

5. A.S. Alzahrani, N. Mukhtar, A. Aljomaiah, H. Aljamei, A. Bakhsh, N. Alsudani et al. The impact of COVID-19 viral 
infection on the hypothalamic-pituitary-adrenal axis. Endocr. Pract. 27, 83-9 (2021)

6. O. Villard, D. Morquin, N. Molinari, I. Raingeard, N. Nagot, J.P. Cristol et al. The plasmatic aldosterone and C-reactive protein levels, and the severity of covid-19: the Dyhor-19 study. J. Clin. Med. 21, 2315 (2020)

7. M. Rieder, L. Wirth, L. Pollmeier, M. Jeserich, I. Goller, N. Baldus et al. Serum ACE2, angiotensin II, and aldosterone levels are unchanged in patients with COVID-19. Am. J. Hypertens. 34, 278-81 (2021)

8. B. Kumar, M. Gopalakrishnan, M.K. Garg, P. Purohit, M. Banerjee, P. Sharma et al. Endocrine dysfunction among patients with COVID-19: a single-center experience from a tertiary hospital in India. Indian J. Endocrinol. Metab. 25, 14-9 (2021)

9. Y. Tan, W. Zhang, Z. Zhu, N. Qiao, Y. Ling, M. Guo et al. Integrating longitudinal clinical laboratory tests with targeted proteomic and transcriptomic analyses reveal the landscape of host responses in COVID-19. Cell Discov. 7, 42 (2021)

10. I. Campi, I. Bulgarelli, A. Dubini, G.B. Perego, E. Tortorici, C. Torlasco et al. The spectrum of thyroid function tests during hospitalization for SARS COV-2 infection. Eur. J. Endocrinol. 184, 699-709 (2021)

11. C. Garbers, S. Heink, T. Korn, S. Rose-John, Interleukin-6: designing specific therapeutics for a complex cytokine. Nat. Rev. Drug Discov. 17, 395-412 (2018)

12. E.H. Choy, F. De Benedetti, T. Takeuchi, M. Hashizume, M.R. John, T. Kishimoto, Translating IL-6 biology into effective treatments. Nat. Rev. Rheumatol. 16, 335-45 (2020)

13. S.A. Jones, J. Scheller, S. Rose-John, Therapeutic strategies for the clinical blockade of IL-6/gp130 signaling. J. Clin. Investig 121, 3375-83 (2011)

14. I. Giannakodimos, G.V. Gkountana, D. Lykouras, K. Karkoulias, $\mathrm{S}$. Tsakas, The role of Interleukin-6 in the pathogenesis, prognosis and treatment of severe COVID-19. Curr. Med. Chem. 28, 5328-5338 (2021)

15. D.E. Leisman, L. Ronner, R. Pinotti, M.D. Taylor, P. Sinha, C.S. Calfee et al. Cytokine elevation in severe and critical COVID-19: a rapid systematic review, meta-analysis, and comparison with other inflammatory syndromes. Lancet Respir. Med. 8, 1233-44 (2020)

16. P. Sabaka, A. Koscalova, I. Straka, J. Hodosy, R. Liptak, B. Kmotorkova et al. Role of interleukin 6 as a predictive factor for a severe course of Covid-19: retrospective data analysis of patients from a long-term care facility during Covid-19 outbreak. BMC Infect. Dis. 21, 308 (2021)

17. R.M.G. Taher Al Barzin, S. Ghafour Raheem, P.K. Khudhur, R. Abdulkarimi, E. Mohammadnejad, A. Tabatabaee, Interleukin-6 role in the severity of COVID-19 and intensive care unit stay length. Cell Mol. Biol. (Noisy-le-grand) 66, 15-8 (2020)

18. C. Tsigos, D.A. Papanicolaou, R. Defensor, C.S. Mitsiadis, I. Kyrou, G.P. Chrousos, Dose effects of recombinant human interleukin- 6 on pituitary hormone secretion and energy expenditure. Neuroendocrinology 66, 54-62 (1997)

19. D.J. Torpy, S.R. Bornstein, G.P. Chrousos, Leptin and interleukin-6 in sepsis. Horm. Metab. Res. 30, 726-9 (1998)

20. G. Mastorakos, G.P. Chrousos, J.S. Weber, Recombinant interleukin-6 activates the hypothalamic-pituitary-adrenal axis in humans. J. Clin. Endocrinol. Metab. 77, 1690-4 (1993)

21. G. Mastorakos, J.S. Weber, M.A. Magiakou, H. Gunn, G.P. Chrousos, Hypothalamic-pituitary-adrenal axis activation and stimulation of systemic vasopressin secretion by recombinant interleukin-6 in humans: potential implications for the syndrome of inappropriate vasopressin secretion. J. Clin. Endocrinol. Metab. 79, 934-9 (1994)

22. E. Spath-Schwalbe, J. Born, H. Schrezenmeier, S.R. Bornstein, P. Stromeyer, S. Drechsler et al. Interleukin-6 stimulates the hypothalamus-pituitary-adrenocortical axis in man. J. Clin. Endocrinol. Metab. 79, 1212-4 (1994)

23. J.C. Pruessner, C. Kirschbaum, G. Meinlschmid, D.H. Hellhammer, Two formulas for computation of the area under the curve represent measures of total hormone concentration versus time-dependent change. Psychoneuroendocrinology 28, 916-31 (2003)

24. G. Path, S.R. Bornstein, M. Ehrhart-Bornstein, W.A. Scherbaum, Interleukin- 6 and the interleukin- 6 receptor in the human adrenal gland: expression and effects on steroidogenesis. J. Clin. Endocrinol. Metab. 82, 2343-9 (1997)

25. G. Path, W.A. Scherbaum, S.R. Bornstein, The role of interleukin6 in the human adrenal gland. Eur. J. Clin. Invest 30(Suppl 3), 91-5 (2000)

26. Y. Mao, B. Xu, W. Guan, D. Xu, F. Li, R. Ren et al. The adrenal cortex, an underestimated site of SARS-CoV-2 infection. Front. Endocrinol. 11, 593179 (2020)

27. V.A. Zinserling, N.Y. Semenova, A.G. Markov, O.V. Rybalchenko, J. Wang, R.N. Rodionov et al. Inflammatory Cell Infiltration of Adrenals in COVID-19. Horm. Metab. Res. 52, 639-41 (2020)

28. W. Kanczkowski, K. Evert, M. Stadtmuller, M. Haberecker, L. Laks, L.S. Chen, K. Frontzek, J. Pablik, C. Hantel, F. Beuschlein et al. COVID-19 targets human adrenal glands. Lancet Diabetes Endocrinol. 21, 00291-6 (2021)

29. M. Freire Santana, M.G.S. Borba, D.C. Baia-da-Silva, F. Val, M. A.A. Alexandre, J.D. Brito-Sousa, G.C. Melo, M.V.O. Queiroga, M.E. Leao Farias, C.C. Camilo et al. Case Report: Adrenal Pathology Findings in Severe COVID-19: an autopsy study. Am. J. Trop. Med Hyg. 103, 1604-1607 (2020)

30. A.C. Iuga, C.C. Marboe, M.Y. M, J.H. Lefkowitch, C. Gauran, S. M. Lagana, Adrenal vascular changes in COVID-19 autopsies. Arch. Pathol. Lab Med. 144, 1159-1160 (2020)

31. R. Pal, M. Banerjee, S.K. Bhadada, Cortisol concentrations and mortality from COVID-19. Lancet Diabetes Endocrinol. 8, 809 (2020)

32. A.H. Hamrahian, T.S. Oseni, B.M. Arafah, Measurements of serum free cortisol in critically ill patients. N. Engl. J. Med. 350, 1629-38 (2004)

33. B. Gibbison, D.M. Keenan, F. Roelfsema, J. Evans, K. Phillips, C. A. Rogers et al. Dynamic pituitary-adrenal interactions in the critically Ill after cardiac surgery. J. Clin. Endocrinol. Metab. 105, 1327-42 (2020)

34. K.W. Choy, Cortisol concentrations and mortality from COVID19. Lancet Diabetes Endocrinol. 8, 808 (2020)

35. R. Laguna-Goya, A. Utrero-Rico, P. Talayero, M. Lasa-Lazaro, A. Ramirez-Fernandez, L. Naranjo et al. IL-6-based mortality risk model for hospitalized patients with COVID-19. J. Allergy Clin. Immunol. 146, 799-807 e9 (2020)

36. K.E. Bethin, S.K. Vogt, L.J. Muglia, Interleukin-6 is an essential, corticotropin-releasing hormone-independent stimulator of the adrenal axis during immune system activation. Proc. Natl Acad. Sci. USA 97, 9317-22 (2000)

37. M.N. Silverman, A.H. Miller, C.A. Biron, B.D. Pearce, Characterization of an interleukin-6- and adrenocorticotropin-dependent, immune-to-adrenal pathway during viral infection. Endocrinology 145, 3580-9 (2004)

38. D. Franchimont, G. Bouma, J. Galon, G.W. Wolkersdorfer, A. Haidan, G.P. Chrousos, S.R. Bornstein, Adrenal cortical activation in murine colitis. Gastroenterology 119, 1560-8 (2000)

39. M.G. Filippa, M.G. Tektonidou, A. Mantzou, G.A. Kaltsas, G.P. Chrousos, P.P. Sfikakis. Adrenocortical dysfunction in rheumatoid arthritis: Alpha narrative review and future directions. Eur. J. Clin. Investig. 52, e13635 (2021).

40. V.C. Veiga, J. Prats, D.L.C. Farias, R.G. Rosa, L.K. Dourado, F. G. Zampieri et al. Effect of tocilizumab on clinical outcomes at 
15 days in patients with severe or critical coronavirus disease 2019: randomised controlled trial. BMJ 372, n84 (2021)

41. N.C. Nicolaides, E. Charmandari, T. Kino, G.P. Chrousos, Stress-related and circadian secretion and target tissue actions of glucocorticoids: impact on health. Front. Endocrinol. 8, 70 (2017)

42. S.E. Sephton, R.M. Sapolsky, H.C. Kraemer, D. Spiegel, Diurnal cortisol rhythm as a predictor of breast cancer survival. J. Natl Cancer Inst. 92, 994-1000 (2000)

43. S.E. Sephton, E. Lush, E.A. Dedert, A.R. Floyd, W.N. Rebholz, F. S. Dhabhar et al. Diurnal cortisol rhythm as a predictor of lung cancer survival. Brain Behav. Immun. 30(Suppl:S1), 63-70 (2013)
44. A. Schrepf, P.H. Thaker, M.J. Goodheart, D. Bender, G.M. Slavich, L. Dahmoush et al. Diurnal cortisol and survival in epithelial ovarian cancer. Psychoneuroendocrinology 53, 256-67 (2015)

45. S. Anesiadou, G. Makris, M. Michou, P. Bali, I. Papassotiriou, F. Apostolakou et al. Salivary cortisol and alpha-amylase daily profiles and stress responses to an academic performance test and a moral cognition task in children with neurodevelopmental disorders. Stress Health 37, 45-59 (2021)

46. E. Angeli, T. Korpa, E.O. Johnson, F. Apostolakou, I. Papassotiriou, G.P. Chrousos et al. Salivary cortisol and alpha-amylase diurnal profiles and stress reactivity in children with Attention Deficit Hyperactivity Disorder. Psychoneuroendocrinology 90, 174-81 (2018) 